\title{
Neuroenteric cyst of the optic nerve: case report with immunohistochemical study
}

\author{
F Scaravilli, H Lidov, D J Spalton, L Symon
}

\begin{abstract}
A 36 year old man developed slowly progressive unilateral visual loss due to a cystic lesion of the intraorbital optic nerve. Pathologically the lesion was an epithelium lined cyst entirely within the atrophic nerve. The cyst lining consisted of columnar epithelium partly pseudostratified and ciliated with evidence of mucin secretion, and was immunoactive for cytokeratin but not glial fibrillary acidic protein. This lesion is considered to be similar to neuroenteric cysts that have been reported elsewhere in the nervous system, and the immunocytochemical results are consistent with a heterotopia derived from the primitive ectoderm of the stomatodeum.
\end{abstract}

$(\mathcal{F}$ Neurol Neurosurg Psychiatry 1992;55:1197-1199)

Cystic lesions of the optic nerve are rare. They can occur in association with gliomas or meningiomas, from extension of a craniopharyngioma or from the arachnoid. A variety of cysts with an epithelial lining have been described in the nervous system in many locations ${ }^{124671218-202425}$ but there has been only one report of a cyst of this type in the optic nerve. ${ }^{10}$ The classification of these cysts, and hypotheses about their origin have depended on their histology and their location. This case is of an epithelial cyst within the optic nerve. The cyst was removed in its entirety, extensively studied histologically and characterised by immunocytochemical staining.

Departments of Neuropathology and Neurosurgery, National Hospital for

Neurology and

Neurosurgery,

London, UK

F Scaravilli

H Lidov

L Symon

Medical Eye Unit, St Thomas Hospital, London, UK

D J Symon

Correspondence to:

Dr Scaravilli, Department of Neuropathology, Institute of Neurology, Queen Square,

London WCIN 3BG, UK

Received 7 November 1991 and in revised form

and in revised

Accepted 24 April 1992

\section{Case report}

The patient presented at the age of 19 when he was noted to have a visual acuity (VA) of $6 / 9$ in the left eye and a pale optic disc. He attended irregularly for follow up. Fourteen years later the VA had fallen to $6 / 12$. He declined investigation and was seen again two years later, now at 36 years, at which point his vision had fallen to $6 / 36$. At this time he complained of progressive blurred vision over the previous 2 years and left frontal headaches. His past medical history and family history were unremarkable.

On examination the right eye was entirely normal. The visual acuity had decreased to $6 / 36$ in the left eye, with a small central area of preserved field. The optic disc on the left was pale. The remainder of the neurological examination was normal. CT (fig 1) showed a well defined intrinsic mass enlarging the posterior intraorbital portion of the optic nerve which was felt to represent either a glioma or meningioma. $T_{1}$ weighted $M R I$ scans confirmed this. An orbito-cranial exploration was performed and the optic nerve inspected. The posterior orbital optic nerve was distended and cystic, partly extending into the optic canal and, on incision, a milky fluid was released. The affected optic nerve was totally excised leaving the dura intact. Postoperatively the patient recovered uneventfully.

\section{Pathological examination}

The surgical specimen consisted of $2 \mathrm{~cm}$ of optic nerve measuring $2.5 \mathrm{~mm}$ in diameter. This appeared normal, presumably due to decompression of the intraneural cyst at surgery, and the gross appearance was unremarkable. The tissue was embedded in paraffin and transverse and longitudinal sections were stained with routine methods. Immunocytochemical staining for glial fibrillary acidic protein (GFAP, Dako Ltd. UK) carcinoembryonic antigen (CEA, Dako Ltd, UK), cytokeratin (CAM 5.2 Becton Dickinson, USA), epithelial membrane antigen (EMA, Dako Ltd, UK), neuron specific enolase (NSE), (BAKO Ltd, UK) and synaptophysin (Dako Ltd, UK) was performed using the avidinbiotin method. CAM 5.2 recognizes keratin subgroup 8,18 and 19. ${ }^{11}$ On microscopic examination an irregular slitlike cavity extended to much of the length of the nerve (fig 2a); the cyst occupied almost the whole width of the nerve, without reaching its edges. The lining of the cyst varied from columnar to pseudostratified epithelium, the latter with cilia in most areas, which covered a thin but well developed matrix of collagen fibrils. These were highlighted by reticulin stains, but were PAS negative. With PAS staining it was also apparent that there were a few scattered mucin secreting goblet cells. No evidence of squamous differentiation suggestive of a dermoid or epidermoid cyst and no mitotic figures were seen. The epithelium stained weakly with CEA, and intensely with cytokeratin (fig 2b) but did not react with EMA, NSE, synaptophysin or GFAP, although gliotic tissue surrounding the cyst was immunoreactive for the latter. The surrounding nerve contained only a few myelinated axons along one edge, and was for the rest largely devoid of myelin sheaths. 


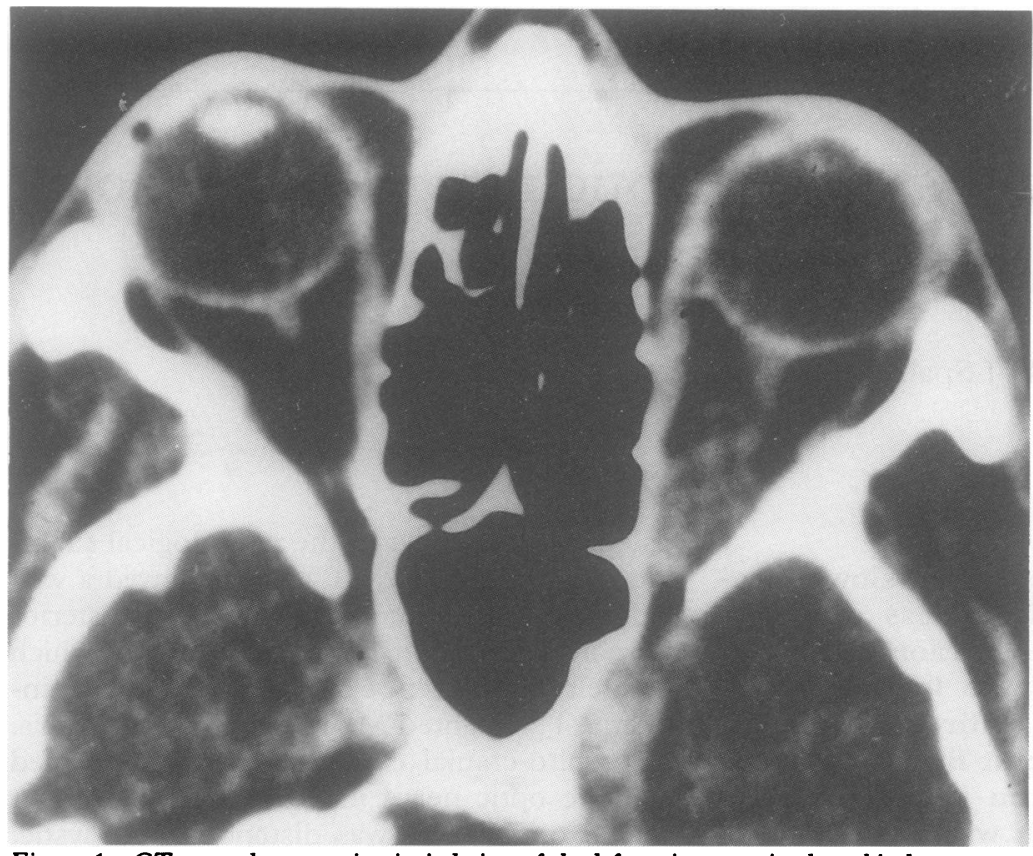

Figure 1 CT scan shows an intrinsic lesion of the left optic nerve in the orbital apex.
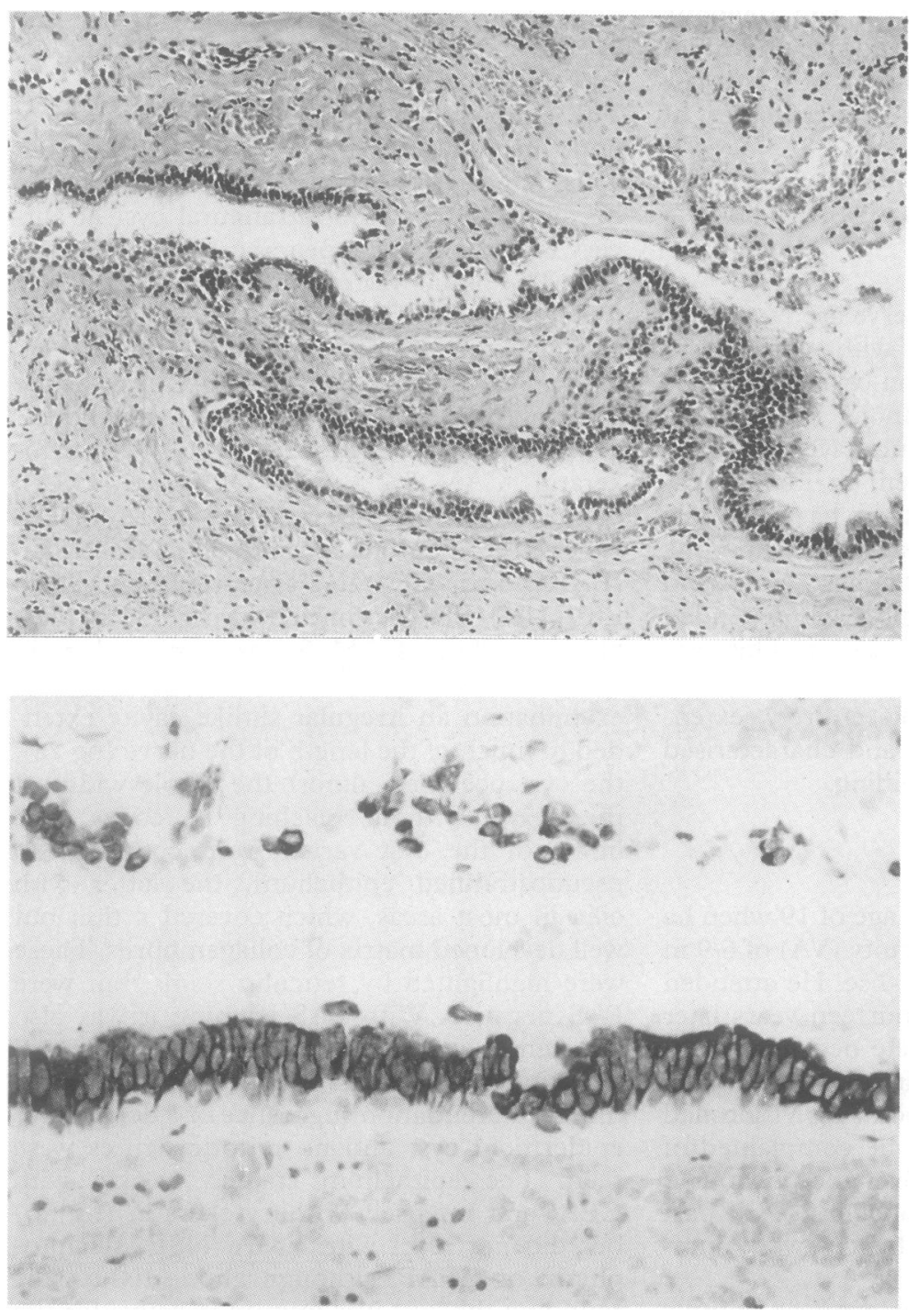

Figure 2a (left) and b (right) Photomicrographs of a transverse section of the optic nerve (a left) showing the cystic cavity. Because of its irregularity, this appears to have 2 separate lumina. $H$. $\mathcal{E} E \times 120$. Photomicrograph (b right) showing the intense immunostaining of the epithelium of the cyst for cytokeratin. $\times 300$.

\section{Discussion}

Cystic lesions of the optic nerve are infrequently described in the literature. ${ }^{9}$ Examples have been reported in conjunction with neoplasms, particularly craniopharyngiomas ${ }^{22}$ involving the chiasm, and gliomas, ${ }^{31721}$ instances in which the cystic lesion is clearly an extension or part of the tumour. Meningiomas may produce cystic dilatation of the subarachnoid space around the optic nerve presumably by trapping CSF. ${ }^{13}$ Cystic lesions have also been described arising from the coverings of the optic nerve, the so called arachnoid cysts ${ }^{814}$ which are extrinsic to the nerve. Recently a single case, similar to the present one was described as a neuroepithelial cyst of the optic nerve and studied with routine histological methods. ${ }^{10}$

Cysts lined by a mucus secreting ciliated columnar epithelium have been found throughout the nervous system, ${ }^{124671218-202425}$ and named according to the location. Thus lesions essentially indistinguishable on histological grounds have been referred to as neuroenteric cysts in the spinal cord and posterior fossa ${ }^{168}$ neuroepithelial cysts in the hemispheres, ${ }^{41215}$ colloid cysts of the third ventricle ${ }^{2022}$ and Rathke cleft cysts in the sella. ${ }^{251925}$ The histological features at each of these locations cover a similar spectrum but are variable and non-specific. Typical cysts are lined by a columnar epithelium with transition to low cuboidal and heaped up to a pseudostratified pattern in other areas covering a collagenous basement membrane. Cilia may be present and, when abundant, the appearances of the epithelium are similar to those of respiratory mucosa; this similarity is further enhanced by the presence of mucus secreting PAS positive goblet cells. Ultrastructural studies have not revealed any features which reliably distinguish cysts at one location from any other. ${ }^{35122122-24}$

The pathological features of the cyst in this report fit the general pattern. The possible origin of such cystic lesions has been variously attributed in relationship to the particular location in which each occurs. It has been suggested that they are trapped embryonic remnants - thus enteric remnants in the spinal cord, ${ }^{5}$ ependymal remnants in the forebrain $^{451520}$ and stomodeal remnants in the hypophysis. ${ }^{21825}$ Results from a retrospective unpublished study of 7 "neurenteric cysts" of the spinal cord and posterior fossa and 3 hemispheric colloid cysts indicate a pattern of staining similar to that seen in the present lesion, that is, reactivity for either cytokeratin or EMA, and uniform absence of GFAP.

The occurrence of an epithelial lined cyst in the optic nerve poses some difficulty from an embryological point of view. Arguments based on embryonic connections, between the neural tube and mesoderm or endoderm, ${ }^{67}$ do not apply at this site. The location seems an implausible one for remnants of the gut, the choroid plexus, ${ }^{4}$ the stomodeum, ${ }^{25}$ or the paraphysis. ${ }^{20}$ Since the eye and the anlage of the optic nerve develop as an outpouching of the diencephalic neural tube, the entrapment 
of primordial ependyma or neuroepithelium seems most likely. Although NSE antibody is not regarded as absolutely reliable, the negative results obtained with it as well as with synaptophysin, rules out the neuroepithelial nature of the epithelium of the cyst. Moreover, there are also obvious differences between the mature ependymal lining and the lining of this cyst. The mature ependyma is usually a simple nonciliated cuboidal epithelium and, like the epithelium in this specimen, does not have a basement membrane. Immature and reactive ependyma as well as ependymal neoplasms are reactive for GFAP but normal mature ependyma is not. ${ }^{16}$ Immunocytochemical staining was performed with this question in mind. The cyst epithelium in the present case was nonreactive for GFAP, which is difficult to reconcile with a trapped remnant of the ependymal lining; furthermore, the reactivity for CAM 5.2 suggests a relationship to an endodermal epithelium, although the reactivity of ependyma with this marker has not been fully explored. ${ }^{16}$

It is clear that in our case there are significant differences between cells derived from the ependyma and the lining of the cyst. The question remains whether or not these can be explained by the degree of maturation and influence of the surrounding tissues or whether the origin of the cells in this case comes from ectopic stomatodeum of Rathke's pouch. Whilst the epithelial characteristics of the cyst would certainly be compatible with an endodermal origin, the method of origin within the optic nerve remains to be explained.

We are grateful to Professor A Garner, Moorfield's Eye Hospital, for helpful discussion, and to Mrs Patricia Jagger for technical assistance.

1 Anderes JP. Neurenteric cysts of the spinal cord and brainstem, MD Thesis, Lausanne, 1984

2 Barry RG, Schlezinger NS, Rathke. Cleft Cysts. Arch Neurol
1959;1:62-72

3 Brihaye M, Graff G, Brihaye J, David P. Volumineux gliome kystique tu chiasma a symptomatologie cerebrale predominants. Acta Neuro et Psych (Belg) 1961;61:525-38.

4 Friede RL, Yasargil MGI. Supratentorial intracranial epithelial (ependymal) cysts; review, case reports and fine structure. $\mathcal{f}$ Neurol Neurosurg Psychiatry 1977;40: structure.

5 Ghatak NR, Hirano A, Ksloff SS, Zimmerman HM. Fine structure of an intracerebral epithelial cyst. $f$ Neurosurg 1974;41:75-82.

6 Harriman DGF. An Intraspinal Enterogenous Cyst. $\mathcal{f}$ Path Bact 1958;75:413-19.

7 Hirai O, Kawamura J, Kukumitsu T. Prepontine epitheliumlined cysts. F Neurosurg 1981;55:312-7.

8 Hirano A, Ghatak NR, Wisoff HS, Zimmerman HM. An Epithelial Cyst of the Spinal Cord. An Electron Microscopic Study. Acta Neuropath (Berl) 1971;18:214-23.

9 Holt $\mathrm{H}$. Cysts of the intracranial portion of the optic nerve. Am $\mathscr{f}$ Opthalmol 1966;61:1166-70.

10 Isla A, Palacios J, Roda JM, Gutierrez M, Gonzalez C, Blazquez MG. Neuroepithelial cyst in the optic nerve: case report. $\mathcal{F}$ Neurosurg 1987;67:137-9.

11 Johnson D E, Herudier BG, Medeiros LJ, Waruke RA, Rouse RV. The diagnostic utility of keratin profiles of hepatocellular carcinoma and cholangiocarcinoma. $A m \mathcal{F}$ Surg Pathol 1988;12:187-97.

12 Markwalder TM, Zimmerman A. Intracerebral Ciliated Epithelial Cysts. Surg Neurol 1979;11:195-8.

$13 \mathrm{McNab}$ AA, Wright JE. Cysts of the optic nerve: three cases associated with meningioma. Eye 1989;3:355-9.

14 Miller NR, Green WR. Arachnoid cysts involving a portion of the intraorbital optic nerve. Arch Opthal 1975;93: $1117-21$.

15 Palma L. Supratentorial neuroepithelial cysts: Report of two cases. I Neurosurg 1975;42:353-7.

16 Perentes E, Rubenstein LI. Recent applications of immunoperoxidaso histochemistry in human neuro-oncology. peroxidaso histochemistry in human
Arch Pathol Lab Med 1987;111:796-812.

17 Reese A. Glioma of the optic nerve, retina and orbit. Tumors of the eye. Hagerstown: Harper and Row 1976 134-45.

18 Ringel SP, Bailey OT. Rathke's cleft cysts. $f$ Neurol Neurosurg Psychiatry 1972;35:693-7.

19 Shuangshoti S, Netsky MG, Nashold BS. Epithelial cysts related to sella turicia: Proposed origin from neuroepithelium. Arch Path 1970;90:444-50.

20 Shuangshoti S, Roberts MP, Netsky MG. Neuroepithelial (colloid) cysts. Arch Path 1965;80:214-24.

21 Spencer WH. Primary neoplasms of the optic nerve and sheath. Tr Am Opth Soc 1972;70:490-528.

22 Walsh FB, Chambers JW, Lloyd LA. The ocular signs of tumour involving the anterior visual pathways. Am $\mathfrak{f}$ Ophthalmol 1956;42:347-77.

23 Yagishita S, Itoh Y, Shiozowa T, Tanaka T. Ultrastructural observation on a colloid cyst of the third ventricle: A contribution to its pathogenesis. Acta Neuropathol (Berl) 1984;65:41-5.

24 Yoshida T, Hakatani S, Shimizu K, Yamada K, Yukitak U, Heitaro M. Huge epithelium-lined cysts: report of two cases. F Neurol Neurosurg Psych 1986;49:1458-9.

25 Yoshida J, Kobayashi T, Kageyama N, Kanzaki M. Symptomatic Rathke's cleft cyst: Morphological study with light and electron microscopy and tissue culture $\mathcal{F}$ Neurosurg 1977;47:451-8. 